This is the author's final, peer-reviewed manuscript as accepted for publication. The publisher-formatted version may be available through the publisher's web site or your institution's library.

\title{
Factors affecting sperm recovery rates and survival after centrifugation of equine semen
}

M. S. Ferrer, S. K. Lyle, B. E. Eilts, A. H. Eljarrah, D. L. Paccamonti

\section{How to cite this manuscript}

If you make reference to this version of the manuscript, use the following information:

Ferrer, M. S., Lyle, S. K., Eilts, B. E., Eljarrah, A. H., \& Paccamonti, D. L. (2012). Factors affecting sperm recovery rates and survival after centrifugation of equine semen. Retrieved from http://krex.ksu.edu

\section{Published Version Information}

Citation: Ferrer, M. S., Lyle, S. K., Eilts, B. E., Eljarrah, A. H., \& Paccamonti, D. L. (2012). Factors affecting sperm recovery rates and survival after centrifugation of equine semen. Theriogenology, 78(8), 1814-1823.

Copyright: (c) 2012 Elsevier Inc.

Digital Object Identifier (DOI): doi:10.1016/j.theriogenology.2012.07.011

Publisher's Link: http://www.theriojournal.com/article/S0093-691X\%2812\%29004116/abstract

This item was retrieved from the K-State Research Exchange (K-REx), the institutional repository of Kansas State University. K-REx is available at http://krex.ksu.edu 
1 Factors affecting sperm recovery rates and survival after centrifugation of equine semen

2

$5 \quad{ }^{a}$ Equine Health Studies Program, Department of Veterinary Clinical Sciences, Louisiana State

$8 \quad{ }^{*}$ Corresponding author and present address at: Department of Clinical Sciences, College of

9 Veterinary Medicine, Kansas State University, Manhattan, KS 66506, USA. Tel.: +1-785-532-

10 5700. Fax: +1-785-532-4989. Email address: mferrer@vet.k-state.edu

11

$12{ }^{1}$ Present address: Department of Veterinary Clinical Sciences, Jordan University of Science and

13 Technology, Irbid, Jordan

14

15

16

17

18

19

20

21 
Abstract

Conventional centrifugation protocols result in important sperm losses during removal of

25 the supernatant. In this study, the effect of centrifugation force (400 or $900 \times \mathrm{g}$ ), duration (5 or

$2610 \mathrm{~min})$ and column height $(20$ or $40 \mathrm{~mL})($ Exp. 1$)$; sperm concentration $(25,50$ and $100 \mathrm{x}$

$2710^{6} / \mathrm{mL}$; Exp. 2) and centrifugation medium (EZ-Mixin CST, INRA96 or VMDZ; Exp. 3) on

28 sperm recovery and survival after centrifugation and cooling and storage was evaluated. Overall,

29 sperm survival was not affected by the combination of centrifugation protocol and cooling. Total

30 sperm yield (TY) was highest after centrifugation for $10 \mathrm{~min}$ at $400 \mathrm{x}$ g in 20 -mL columns $(95.6$

$31 \pm 5 \%)$ or $900 \times \mathrm{g}$ in $20-(99.2 \pm 0.8 \%)$ or $40-\mathrm{mL}(91.4 \pm 4.5 \%)$ columns, and at $900 \mathrm{x}$ g for 5

$32 \mathrm{~min}$ in $20-\mathrm{mL}$ columns $(93.8 \pm 8.9 \%)(\mathrm{P}<0.0001)$. Total $(\mathrm{TMY})$ and progressively motile

33 (PMY) sperm yield followed a similar pattern $(\mathrm{P}<0.0001)$. Sperm yields were not significantly

34 different among samples centrifuged at different sperm concentrations. However, centrifugation

35 at $100 \times 10^{6} / \mathrm{mL}$ resulted in significantly lower TY $(83.8 \pm 10.7 \%)$ and TMY $(81.7 \pm 6.8 \%)$

36 compared with non-centrifuged semen. Centrifugation in VMDZ resulted in significantly lower

37 TMY $(69.3 \pm 22.6 \%)$, PMY $(63.5 \pm 18.2 \%)$, viable yield $(60.9 \pm 36.5 \%)$ and survival of

38 progressively motile sperm after cooling $(21 \pm 10.8 \%)$ compared with non-centrifuged semen.

39 In conclusion, centrifuging volumes of $\leq 20 \mathrm{~mL}$ minimized sperm losses with conventional

40 protocols. With 40-mL columns, it may be recommended to increase the centrifugal force to 900

$41 \mathrm{xg}$ for $10 \mathrm{~min}$ and dilute the semen to a sperm concentration of 25 to $50 \times 10^{6} / \mathrm{mL}$ in a milk- or

42 fractionated milk-based medium. The semen extender VMDZ did not seem well suited for

43 centrifugation of equine semen. 
45 Keywords: Centrifugation, stallion, semen, viability, recovery

1. Introduction

Equine semen is routinely centrifuged prior to cryopreservation to concentrate sperm and

50 minimize the adverse effects of seminal plasma on post-thaw motility $[1,2]$. Depending on the

51 semen extender used, centrifugation and partial removal of seminal plasma prior to cooling may

52 also be beneficial for sperm motility, and acrosome and DNA integrity, especially for stallions

53 whose sperm suffer a significant decrease in motility when processed in a conventional manner

54 by simple dilution of seminal plasma with semen extender [3-6]. Ejaculates with low sperm

55 concentration require centrifugation to allow adequate dilution of semen for cooling [7].

In conventional centrifugation protocols, equine semen is diluted 1:1 (v:v) or to a sperm

57 concentration of $50 \times 10^{6} / \mathrm{mL}$ in a milk-based semen extender for centrifugation. A $40-\mathrm{mL}$

58 volume of extended semen is typically loaded into $50-\mathrm{mL}$ conical tubes, and centrifuged at 400

59 to $600 \mathrm{x}$ g for 10 to $15 \mathrm{~min}$ [7]. After centrifugation, $30 \mathrm{~mL}$ of the supernatant is removed,

60 retaining 5 to $20 \%$ of seminal plasma in the resuspended sample [7]. The final concentration of

61 seminal plasma depends on the amount of semen extender added to the pellet. Around 20 to 25

$62 \%$ of sperm are lost with the supernatant during conventional centrifugation protocols $[7,8]$, with

63 losses of up to $46 \%$ of sperm reported [9]. This results in an important reduction in the number

64 of insemination doses available per ejaculate. A centrifugation protocol that improves sperm

65 recovery, without damaging the cells, would result in a higher number of viable sperm available

66 for cryopreservation or insemination. 
Cushioned centrifugation in optically clear media has been reported to improve recovery

68 rates without detrimental effects on sperm viability compared to conventional centrifugation

69 protocols $[10,11]$. However, the improved recovery rates are likely to result from increased

70 centrifugation duration (20 $\mathrm{min}$ ) and forces (1000 x g) used during cushioned centrifugation [11].

71 In fact, better recovery rates were obtained after centrifugation in an opaque medium at $1000 \mathrm{xg}$

72 for 20 min without an underlaying cushion compared with the addition of a cushion [11]. Use of

73 a cushion to protect equine sperm against damage associated with close packing was previously

74 suggested to be unnecessary [12]. Use of cushioned centrifugation increases the time and

75 expenses associated with centrifuging equine semen. A simpler centrifugation protocol that

76 improves recovery rates without damaging sperm and increasing processing time and expenses

77 would be of benefit for the equine industry.

78 Sedimentation rate, and therefore sperm recovery, is determined by the centrifugal force and

79 duration of centrifugation. Centrifugation duration and force are reciprocal, and total yield

80 increases linearly as the product of duration $\mathrm{x}$ force increases until it reaches full sedimentation

81 at $100 \%[13,14]$. Once full sedimentation is reached, viable and motile yields decrease as a

82 consequence of cell damage in the pellet and the lack of further arrival of undamaged cells

$83[13,14]$. A particle also experiences a greater centrifugal force the further away it is from the axis

84 of rotation. A shorter column height in a partially filled tube increases the minimum radial

85 distance of the particles from the axis of rotation. Therefore, particles start to sediment at a

86 higher gravitational field, have a reduced path length to travel, and sedimentation is quicker [13-

87 15]. Sedimentation rate also depends on the difference in specific gravity between the cells and

88 the surrounding medium, and the viscosity of the medium. This results in an increase in

89 sedimentation rate as the density and viscosity of the medium decrease [13-15]. Initial sperm 
90 concentration differs among ejaculates. Therefore, if an ejaculate is diluted with an equal volume

91 of semen extender for centrifugation [7], semen is centrifuged at different sperm concentrations.

92 While sedimentation rate can be affected by the initial concentration of the cell suspension [15],

93 the effect of sperm concentration on recovery rates after centrifugation has not been critically

94 evaluated. The objectives of this study were to determine the effect of two different centrifugal

95 forces, durations, and column heights (volume), and three different sperm concentrations and

96 media (semen extender) on sperm recovery rate and survival after centrifugation. Since

97 centrifugation is often performed prior to cooling, delayed effects of centrifugation on sperm

98 motility and viability after $24 \mathrm{~h}$ of cold storage at 4 to $8{ }^{\circ} \mathrm{C}$ were also evaluated.

99

100 2. Materials and Methods

101

102 2.1. Stallions and semen collection

103

Semen was collected from seven (Exp. 1 and 2) or five (Exp. 3) light breed adult

105 stallions. Stallions 1 to 7 were used in Exp. 1. Stallions 8 to 14 were used in Exp. 2, while only

106 stallions 8 to 12 were included in Exp. 3. Stallions were housed in individual pens supplemented

107 with a pelleted ration and grass hay at the School of Animal Sciences or the School of Veterinary

108 Medicine, Louisiana State University, Baton Rouge, Louisiana (Exp. 1) or Kansas State

109 University, Manhattan, Kansas (Exp. 2 and 3). The stallions were teased with a mare in estrus

110 and the penis was washed with warm water prior to semen collection. One ejaculate was

111 collected from each stallion for each experiment with a Colorado (Exp. 1) or Missouri (Exp. 2

112 and 3) model artificial vagina over a phantom mare. Semen was obtained in February (Exp. 1), 
113 August (Exp. 2) or September (Exp. 3) from sexually rested stallions. The internal temperature

114 of the artificial vagina was adjusted at 45 to $48{ }^{\circ} \mathrm{C}$, and sterile non-spermicidal lubricant (Priority

115 Care, First Priority Inc., Elgin, IL, USA) was applied in the proximal one third of the artificial

116 vagina immediately before collection. An in-line disposable nylon mesh gel filter (Animal

117 Reproduction Systems, Chino, CA, USA) was used to exclude the gel fraction of the ejaculate.

118 Immediately after collection, water was drained from the Colorado Model artificial vagina, the

119 filter was removed, and the semen samples were transported to the laboratory for processing 120 within 20 min of collection.

122 2.2. Evaluation of sperm concentration, motility and viability

125 was not validated for repeatability in this study, the hemacytometer remains the gold standard for 126 evaluation of sperm concentration [16,17]. Semen was diluted 1:100 in formalin buffered saline

127 and spermatozoa were counted in the central grid of the hemacytometer. Both chambers of the 128 hemacytometer were counted and averaged. If a difference greater than $10 \%$ was found between 129 chambers in the number of sperm counted, the hemacytometer was re-loaded and the sperm 130 count was repeated. Sperm concentration was expressed in million per milliliter. During

131 Experiment 1, sperm in the supernatant were counted using a 1:10 dilution and the sperm count 132 was divided by 10.

133 Sperm motility was evaluated using a computer assisted sperm analyzer (Exp. 1: Sperm 134 Vision, Minitube of America, Verona, WI, USA; Exp. 2 and 3: IVOS, Hamilton Thorn Research, 135 Beverly, MA, USA). The settings of the instrument were: Frames acquired 45, frame rate $60 \mathrm{~Hz}$, 
136 minimum contrast 80 , minimum cell size 3 pixels, straightness cut off $75 \%$, average path

137 velocity cut off $50 \mu / \mathrm{s}$, VAP cut off static cells $20 \mu / \mathrm{s}$, cell intensity 106 , static size gates 0.38 to

1382.99 , static intensity gates 0.77 to 1.4 , and static elongation gates 12 to 97 . Semen was placed in

139 a $20-\mu \mathrm{L}$ sperm analysis chamber (Hamilton Thorn Research) over the internal heated specimen

140 stage at $37^{\circ} \mathrm{C}$. Mean percentages of total and progressive motility were assessed from 15 fields

141 with a X 10 phase-contrast objective.

142 Membrane integrity or viability was evaluated with a fluorescent probe (SYBR14/PI,

143 Live/Dead Kit, Molecular Probes, Eugene, OR, USA). First, $2 \mu \mathrm{L}$ of a working solution of

144 SYBR14 were added to $400 \mu \mathrm{L}$ of semen. Semen was incubated for $10 \mathrm{~min}$ at $37^{\circ} \mathrm{C}$ in the dark.

145 Then, $2 \mu \mathrm{L}$ of propidium iodide was added and semen was incubated for $10 \mathrm{~min}$ at $37^{\circ} \mathrm{C}$ in the

146 dark. Semen was evaluated using an epifluorescence microscope at high power (X 40)

147 (Olympus B-Max 60, Olympus America, Inc., Melville, NY, USA). One hundred spermatozoa

148 were classified as live or membrane-intact (green fluorescent), or dead or membrane-damaged

149 (red fluorescent). Moribund sperm (combination of green and red fluorescence) were classified

150 as membrane-damaged.

\section{2.3. Semen processing}

Immediately after collection, a standard semen evaluation was performed. Each ejaculate

155 was then divided into aliquots as described below for each experiment. After adding pre-warmed

156 semen extender, and immediately before centrifugation, sperm concentration, motility and

157 membrane integrity were evaluated. Then, the aliquots were centrifuged as described below for 
158 each experiment. Centrifugation duration included the time for rotor acceleration. An immediate

159 breaking feature was not used. The deceleration curve was the same for all treatments.

160 After centrifugation, $37 \mathrm{~mL}$ (40-mL suspensions) or $17 \mathrm{~mL}$ (20-mL suspensions) of the

161 supernatant was removed by aspiration with a $2-\mathrm{mL}$ plastic transfer pipette. Transfer pipettes are

162 readily available and routinely used in andrology laboratories for aspiration of the supernatant.

163 Given the duration and forces used for centrifugation here, a tight pellet was obtained. The

164 supernatant was also removed immediately after centrifugation with minimal time delay.

165 Therefore, sperm loss in the supernatant due to swim up of spermatozoa was unlikely to occur.

166 Sperm concentration was evaluated in the supernatant with a hemacytometer $[8,18]$ and semen

167 extender was added to re-suspend the pellet to a sperm concentration of $25 \times 10^{6} / \mathrm{mL}$. No

168 attempt was made to maintain the concentration of seminal plasma constant. Instead, semen was

169 processed using a routine protocol for cooling, where the final sperm concentration was taken

170 into account. Sperm motility and membrane integrity were assessed in the re-suspended semen

171 immediately. Re-suspended and non-centrifuged control samples were packaged in plastic bags

172 (Whirl-Pack, Nasco, Fort Atkinson, WI, USA), placed in a passive cooling device (Equitainer,

173 Hamilton Thorn Research, Danver, MA, USA) and stored at approximately $4{ }^{\circ} \mathrm{C}$ for $24 \mathrm{~h}$. After

$17424 \mathrm{~h}$ of cold storage, semen was warmed at $37{ }^{\circ} \mathrm{C}$ for $10 \mathrm{~min}$ and sperm motility and membrane

175 integrity were reassessed.

177 2.4. Experiment 1: Effect of centrifugation force, duration and column height on sperm recovery 178 rate and survival 
181 milk-based semen extender (EZ-Mixin CST ${ }^{\circledR}$, Animal Reproduction Systems). The extended

182 semen was divided into nine aliquots. Each aliquot was centrifuged in a swinging bucket rotor

183 centrifuge (Eppendorf 5804, Hamburg, Germany) at room temperature in a 50-mL conical tube

184 under one of two centrifugation forces ( 400 or $900 \mathrm{x} \mathrm{g}$ ), duration ( 5 or $10 \mathrm{~min}$ ) and volumes (20

185 or $40 \mathrm{~mL})($ Table 1$)$.

186

187 2.5. Experiment 2: Effect of sperm concentration on recovery rate and survival

Each ejaculate $(\mathrm{n}=7)$ was divided into four aliquots and extended with a milk-based

190 semen extender (EZ-Mixin CST ${ }^{\circledR}$, Animal Reproduction Systems) to one of the following sperm

191 concentrations: 1) $25 \times 10^{6} / \mathrm{mL}$, uncentrifuged control; 2) $25 \times 10^{6} / \mathrm{mL}$; 3) $50 \times 10^{6} / \mathrm{mL}$; 4) $100 \times$

$19210^{6} / \mathrm{mL}$. Centrifugation of $40 \mathrm{~mL}$ of each aliquot was performed in a swinging bucket rotor

193 centrifuge (Sorvall ST16, Fisher Scientific Co. LLC, Hanover Park, IL, USA) at room

194 temperature in 50-mL conical tubes at $900 \mathrm{x} \mathrm{g}$ for $10 \mathrm{~min}$. This centrifugal force and duration

195 was chosen since it provided the best sperm yields in Exp. 1. After removing the supernatant,

196 semen extender was added to dilute all aliquots to the same final sperm concentration of $25 \times 10^{6}$

$197 / \mathrm{mL}$.

198

199 2.6. Experiment 3: Effect of centrifugation medium on sperm recovery rate and survival

201 Each ejaculate $(\mathrm{n}=5)$ was divided into three aliquots. Each aliquot was diluted to a 202 sperm concentration of $25 \times 10^{6} / \mathrm{mL}$ with a milk-based (EZ-Mixin $\mathrm{CST}^{\circledR}$, Animal Reproduction 
204 egg yolk-based (VMDZ, Partnar Animal Health, Port Huron, MI, USA) semen extender. Forty

205 milliliters from each aliquot served as a non-centrifuged control sample. Other $40 \mathrm{~mL}$ from each

206 aliquot were centrifuged in a swinging bucket rotor centrifuge (Sorvall ST16, Fisher Scientific

207 Co. LLC) at room temperature in 50-mL conical tubes at $900 \mathrm{x} \mathrm{g}$ for $10 \mathrm{~min}$. Since the goal of

208 this experiment was to test the effect of centrifugation medium on sedimentation rates, all other

209 centrifugation conditions were kept constant to eliminate any confounding effects of changing

210 centrifugation conditions. After removing the supernatant, the corresponding semen extender

211 was added to re-suspend the pellet to a final sperm concentration of $25 \times 10^{6} / \mathrm{mL}$.

213 2.7. Calculation of sperm yields and survival factors

216 centrifugation (TSP) $\left(\mathrm{x} 10^{6}\right)=$ initial sperm concentration $\mathrm{x}$ volume in the tube; Total sperm in

217 the supernatant (TSS) $\left(\mathrm{x} 10^{6}\right)=$ sperm concentration in the supernatant $\mathrm{x}$ volume of the

218 supernatant; Total sperm in the pellet $(\mathrm{TSPe})\left(\mathrm{x} 10^{6}\right)=\mathrm{TSP}-\mathrm{TSS}$; Total yield $(\mathrm{TY})=\mathrm{TSPe} /$

219 TSP $\mathrm{x} 100$; Total motile yield $(\mathrm{TMY})=($ TSPe $\mathrm{x} \%$ total motility post-centrifugation $) /($ TSP $\mathrm{x} \%$

220 total motility pre-centrifugation) $\mathrm{x} 100$; Progressively motile yield $(\mathrm{PMY})=(\mathrm{TSPe} \times \mathrm{\%}$

221 progressive motility post-centrifugation) / (TSP $\mathrm{x} \%$ progressive motility pre-centrifugation) $\mathrm{x}$

222100 ; Viable yield $(\mathrm{VY})=(\mathrm{TSPe} \times \mathrm{x} \%$ viability post-centrifugation $) /(\mathrm{TSP} \times \mathrm{x} \%$ viability pre-

223 centrifugation) $\mathrm{x} 100[8,18]$.

224 Sperm motility and viability after centrifugation were normalized to the initial values, 225 and the normalized variables were called survival factors [13,14]. Survival factor is more likely 
226 to reveal differences between treatments since this variable eliminates the effect of individual

227 variation in initial semen quality on the outcome and assess only the changes in semen quality in

228 response to treatment $[13,14]$. Survival factors were calculated as follows: Survival factor for

229 total motility (SFT) $=\%$ total motility post-centrifugation / $\%$ total motility pre-centrifugation $\mathrm{x}$

230100 ; Survival factor for progressive motility $(\mathrm{SFP})=\%$ progressive motility post-centrifugation /

$231 \%$ progressive motility pre-centrifugation $\mathrm{x} 100$; Survival factor for viability (SFV) $=\%$ viability

232 post-centrifugation / \% viability pre-centrifugation $\times 100$.

233 A similar normalization to values post-centrifugation was done after cooling: Survival

234 factor for total motility at $24 \mathrm{~h}(\mathrm{SFT} 24)=\%$ total motility at $24 \mathrm{~h} / \%$ total motility post-

235 centrifugation $\times 100$; Survival factor for progressive motility at $24 \mathrm{~h}(\mathrm{SFP} 24)=\%$ progressive

236 motility at $24 \mathrm{~h} / \%$ progressive motility post-centrifugation $\mathrm{x} 100$; Survival factor for viability at

$23724 \mathrm{~h}(\mathrm{SFV} 24)=\%$ viability at $24 \mathrm{~h} / \%$ viability post-centrifugation $\mathrm{x} 100$.

239 2.8. Statistical analysis

Sperm yields and survival factors after centrifugation and cooling were tested for normality

242 using the Shapiro-Wilk test. Variables followed a normal distribution. The effect of

243 centrifugation protocol on the response variables (TY, TMY, PMY, VY, SFP, SFT, SFV, SFT24,

244 SFP24, SFV24) was evaluated with ANOVA for repeated measures within storage time

245 (immediately after centrifugation or after cooling). The general linear model procedure of SAS

246 package (SAS Institute, Cary, NC, USA) was used for analysis. The model included the random

247 effect of ejaculate and the fixed effect of treatment. In Exp. 1, each treatment represented a

248 different interaction of centrifugation force, duration and volume. In Exp. 2 and 3, each treatment 
249 represented centrifugation with a different sperm concentration or semen extender, respectively.

250 The control non-centrifuged treatments were also included in the models. If there was a

251 significant treatment effect, pre-determined comparisons were made between treatments using

252 least squares means with a Tukey adjustment of Type I error to 0.05 . Differences were

253 considered significant when $\mathrm{P}<0.05$. All values were expressed as mean $\pm \mathrm{SD}$.

255 3. Results

257 3.1. Experiment 1: Effect of centrifugation force, duration and column height on sperm recovery 258 rate and survival

260 Initial total sperm motility was $78.1 \pm 20.4 \%$, progressive sperm motility was $70.7 \pm 22.4 \%$ 261 and sperm viability was $75.8 \pm 14.9 \%$. There was a significant effect of ejaculate on all variables $262(\mathrm{P}<0.05)$ except TY, TMY and SFV24. After centrifugation, one stallion had a decrease in 263 survival factors, one stallion had an improvement in semen quality, and five stallions had no 264 apparent change.

265 Total sperm yield was greater for non-centrifuged semen $(100 \pm 0 \%)$, semen centrifuged at $266400 \mathrm{x}$ g for $10 \mathrm{~min}$ in a $20-\mathrm{mL}$ suspension $(95.6 \pm 5 \%), 900 \mathrm{x} \mathrm{g}$ for $10 \mathrm{~min}$ in a $40-\mathrm{mL}(91.4 \pm$ $2674.5 \%$ ) or $20-\mathrm{mL}$ suspension $(99.2 \pm 0.8 \%)$ and $900 \mathrm{xg}$ for $5 \mathrm{~min}$ in a $20-\mathrm{mL}$ suspension $(93.8 \pm$ $2688.9 \%$ ) compared with semen centrifuged at $400 \mathrm{x}$ g for $10 \mathrm{~min}$ in a 40 -mL suspension $(74.5 \pm$ $2697.6 \%$ ), $400 \mathrm{xg}$ for $5 \mathrm{~min}$ in a $20-\mathrm{mL}$ suspension $(74.3 \pm 8.6 \%)$ and $900 \mathrm{xg}$ for $5 \mathrm{~min}$ in a $40-$ $270 \mathrm{~mL}$ suspension $(72.6 \pm 9.5 \%$ ), whereas centrifugation at $400 \mathrm{x} \mathrm{g}$ for $5 \mathrm{~min}$ in a $40-\mathrm{mL}$

271 suspension provided the lowest total sperm yield $(47.2 \pm 7.3 \%)(\mathrm{P}<0.0001)$. Total and 
272 progressively motile sperm yields followed a similar pattern $(\mathrm{P}<0.0001)($ Table 1$)$. Viable

273 sperm yield was also highest for non-centrifuged semen (100 $\pm 0 \%)$, semen centrifuged at $900 \mathrm{x}$

$274 \mathrm{~g}$ in $20-\mathrm{mL}$ suspensions for $10 \min (92 \pm 18.5 \%)$ or $5 \min (87.8 \pm 13.1 \%), 400 \mathrm{xg}$ for $10 \mathrm{~min}$ in

275 a $20-\mathrm{mL}$ suspension $(86.9 \pm 14.2 \%)$ and $900 \mathrm{x}$ g for $10 \mathrm{~min}$ in a $40-\mathrm{mL}$ suspension $(84.4 \pm 19.3$

$276 \%)$, and lowest after centrifugation at $400 \mathrm{x}$ g for $5 \mathrm{~min}$ in a $40-\mathrm{mL}$ suspension $(44.5 \pm 8 \%)(\mathrm{P}<$

277 0.0001) (Table 1). Centrifugation protocol had no significant effect on any survival factor after

278 centrifugation and cooling (Table 1).

279

280 3.2. Experiment 2: Effect of sperm concentration on recovery rate and survival

281

282

Initial total sperm motility was $76.6 \pm 10.3 \%$, progressive sperm motility was $37.9 \pm 40.4 \%$

283 and sperm viability was $77.5 \pm 16.8 \%$. None of the variables was affected by ejaculate, except

284 SFT24 $(\mathrm{P}=0.0005)$. While response to centrifugation at 25 and $50 \times 10^{6} / \mathrm{mL}$ was variable

285 among stallions, all stallions had a decrease of $\geq 20 \%$ in SFT24 h when semen was centrifuged

286 at $100 \times 10^{6} / \mathrm{mL}$. Neither TY nor TMY differed among centrifuged samples, however

287 centrifugation at a sperm concentration of $100 \times 10^{6} / \mathrm{mL}$ resulted in significantly lower TY $(\mathrm{P}=$

$2880.0293)$ and TMY $(\mathrm{P}=0.0219)$ compared with non-centrifuged semen (Table 2). Viable yield

289 was not different among centrifuged samples, however centrifugation at all concentrations

290 resulted in significantly lower VY compared with non-centrifuged semen $(\mathrm{P}=0.0003)$ (Table 2).

291 Progressively motile yield was not different among treatments $(P=0.0744)$ (Table 2$)$. None of

292 the survival factors after centrifugation and cooling differed significantly among semen samples

293 centrifuged at different concentrations, or compared with non-centrifuged semen (Table 2). 
3.3. Experiment 3: Effect of centrifugation medium on sperm recovery rate and survival

Initial total sperm motility was $68.7 \pm 13.4 \%$, progressive sperm motility was $36.6 \pm 13.4 \%$

298 and sperm viability was $69.3 \pm 24.9 \%$. There was no significant effect of ejaculate on any of the

299 variables, except SFT24 and SFP24 $(\mathrm{P}=0.0005)$. Total sperm yield was not significantly

300 different among centrifuged samples, but centrifugation in INRA96 resulted in lower TY

301 compared to non-centrifuged semen $(\mathrm{P}=0.0022)$ (Table 3$)$. Total and progressively motile, and

302 viable sperm yield were not significantly different among centrifuged samples. However,

303 centrifugation in VMDZ resulted in lower TMY $(\mathrm{P}=0.0041)$, PMY $(\mathrm{P}=0.0050)$ and VY $(\mathrm{P}=$

304 0.0116) compared to non-centrifuged semen (Table 3). None of the survival factors after

305 centrifugation and cooling differed significantly among treatments, except SFP24. Semen

306 centrifuged in VMDZ had lower progressive motility after cooling compared with its non-

307 centrifuged control sample $(\mathrm{P}=0.0344)$ (Table 3$)$.

308

309 4. Discussion

310

311 The objectives of this study were to identify factors that affected sedimentation rates and

312 survival of equine spermatozoa after centrifugation. Possible delayed effects of centrifugation on

313 sperm function were assessed after $24 \mathrm{~h}$ of cold storage. The motile or viable yield in the pellet

314 and not the percent motility or viability is the parameter that best reflects the effectiveness of a

315 centrifugation protocol [13]. Also, because of the large variability in initial sperm motility and

316 viability among stallions, these parameters were normalized to eliminate this source of variation.

317 The normalized variables were called survival factors [13]. 
318 Survival factors after centrifugation were not affected by treatment in any of the experiments.

319 Furthermore, no delayed effect of centrifugation on sperm motility and viability was evident

320 after cooling for $24 \mathrm{~h}$ with most treatments. Only centrifugation in VMDZ resulted in a decrease

321 in progressive motility after cooling. It can therefore be assumed that, under most of the

322 conditions tested in this study, loss of motile or viable sperm was a result of a decrease in

323 sedimentation rate through the supernatant rather than cell death or damage within the pellet.

324 The rate of sedimentation (v) of a particle is given by the following formula:

$$
v=2 r_{p}^{2}\left(\rho_{p}-\rho_{m}\right) w^{2} r
$$

$$
9 \eta\left(f / f_{\mathrm{o}}\right)
$$

Where, $r_{p}$ is the radius of the particle, $\rho_{p}$ is the density of the particle, $\rho_{m}$ is the density of the

328 medium, $\mathrm{w}$ is the angular velocity of the rotor, $\mathrm{r}$ is the radial distance of the particle from the axis

329 of rotation, $\eta$ is the viscosity coefficient of the medium, $f$ is the frictional coefficient of the

330 hydrated aspherical particle, and $f_{\mathrm{o}}$ is the theoretical frictional coefficient of an unhydrated

331 sphere of the same molecular mass and density $[13,15]$. Therefore, the rotational speed of the

332 rotor, radial distance of the particles from the axis of rotation (given by the column height), and

333 the density and viscosity of the medium affect sedimentation rate. While the radius, density and

334 shape of the particle also affect sedimentation rate, these effects remain constant when

335 comparing centrifugation protocols for a given cell type, such as sperm in the case of this study.

336 As the centrifugal force increases, sedimentation rate also increases. The centrifugal force $(G)$ is

337 given by:

$$
\mathrm{G}=\mathrm{w}^{2} \mathrm{r}
$$

339 Hence, a particle experiences a greater force the further away it is from the axis of rotation. A 340 shorter column height in a partially filled tube increases the minimum radial distance (Fig. 1). 
341 Therefore, particles start to sediment at a higher gravitational field, have a reduced path length to

342 travel, and sedimentation is quicker [13-15].

343 In this study, $28 \%$ of motile and viable sperm were lost with the supernatant after a

344 conventional centrifugation protocol at $400 \mathrm{xg}$ for $10 \mathrm{~min}$ and a volume of $40 \mathrm{~mL}$, which is

345 similar to other reports [7,8]. When the volume of the suspension was reduced to $20 \mathrm{~mL}$,

346 resulting in a shorter column, sperm losses were significantly reduced to $<5 \%$ after

347 centrifugation at a conventional force $(400 \mathrm{x} \mathrm{g})$ and duration $(10 \mathrm{~min})$. Total and viable sperm

348 yields were affected by the height of the suspension.

349 When centrifuging a conventional volume $(40 \mathrm{~mL})$ of semen in a $50-\mathrm{mL}$ tube for a

350 conventional duration (10 min), increasing the centrifugal force to $900 \mathrm{x} g$ also improved sperm

351 yields. Similar increases in sperm recovery rates after increasing centrifugal force were reported

352 previously $[8,9,18]$. Centrifugation duration and force are reciprocal, and total yield increases

353 linearly as the product of duration $\mathrm{x}$ force increases, until it plateaus at $100 \%$. The deleterious

354 effect of centrifugation on sperm function has been attributed to mechanical damage [14], tight

355 packing [14], and production of reactive oxygen species in the pellet [19]. Assuming cells are

356 damaged as a consequence of being packed within the pellet and not of sedimenting through the

357 supernatant, the viable and motile yields depend on the rate at which cells in the pellet are

358 damaged and the rate at which undamaged cells arrive in the pellet $[13,14]$. Once full

359 sedimentation is reached, viable and motile yields decrease as a consequence of cell damage in

360 the pellet and the lack of further arrival of undamaged cells $[13,14]$. Total yield almost reached

361 the plateau at $99 \%$ when semen was centrifuged at $900 \mathrm{x}$ g for $10 \mathrm{~min}$ in 20 -mL suspensions.

362 Increasing the centrifugation duration or force beyond this seemed unnecessary when

363 centrifuging low volumes. Decreasing the centrifugation duration to 5 min resulted in decreased 
364 sperm yields, except when semen was centrifuged in $20-\mathrm{mL}$ suspensions at $900 \mathrm{x} \mathrm{g}$. It seemed

365 then possible to decrease processing time using a higher force with small volumes of semen

366 without compromising recovery rates.

367 At any given centrifugation duration and force, sedimentation rate depends on the difference

368 in specific gravity between the cells and the surrounding medium, and the viscosity of the

369 medium [13-15]. Sedimentation rate increases as the density and viscosity of the medium

370 decrease [15]. Centrifugation medium affected recovery of sperm in this study. Density of the

371 media seemed similar among EZ mixin (1.0125 gr/mL), INRA96 (1.0095 gr/mL) and VMDZ

$372(1.011 \mathrm{gr} / \mathrm{mL})$ semen extenders. However, it is possible that such a small difference in density

373 accounted for differences in sperm recovery. Viscosity of the media was not known and may

374 have been partly responsible for differences in sedimentation also. Centrifugation in INRA96

375 resulted in a significant loss of about $18 \%$ of the initial sperm suspension compared with non-

376 centrifuged samples. However, survival factors for total and progressive motility were $\geq 100 \%$

377 since removing the supernatant and re-suspending the pellet in INRA96 resulted in an

378 improvement in sperm motility in four of the five stallions in this study. The ability of this semen

379 extender to improve sperm motility compensated for the lower sedimentation rate, and resulted

380 in no significant losses of motile sperm. These results cannot be extrapolated to conventional

381 centrifugation protocols. Total sperm yield after centrifugation in INRA96 at $400 \mathrm{x}$ g for $10 \mathrm{~min}$

382 was $54 \%$ [9]. Using a higher centrifugation force may be recommended to minimize sperm

383 losses when using this semen extender.

384 On the other hand, VMDZ seemed unable to protect sperm from immediate and delayed 385 deleterious effects of centrifugation. A significant loss of total (31\%) and progressively motile $386(13 \%)$ sperm occurred after centrifugation in VMDZ compared to non-centrifuged semen. 
387 Centrifugation in VMDZ resulted in an immediate reduction in sperm motility in four of the five

388 stallions in the study. This may have accounted for the decrease in motile sperm yields in spite of

389 the lack of difference in sedimentation rates. Furthermore, there was a dramatic $79 \%$ decrease in

390 progressive sperm motility after cooling semen centrifuged in VMDZ. Centrifugation in VMDZ

391 resulted in a hard pellet that required prolonged pipetting for re-suspension. A loss of sperm

392 motility and membrane integrity was reported after pipetting non-centrifuged rat and mouse

393 sperm [20]. However, there seems to be a species difference in sensitivity of sperm to

394 mechanical damage induced by pipetting since this procedure had no deleterious effects on bull,

395 ram and boar sperm [20]. The effect of pipetting on equine sperm has not been critically

396 evaluated and may have accounted for the immediate or delayed deleterious effects of

397 centrifugation in VMDZ on sperm motility in this study. Also, removal of seminal plasma by

398 centrifugation resulted in lower post-thaw sperm motility and higher lipid peroxidation when

399 buck semen was frozen in an egg yolk-based extender compared with non-centrifuged semen, or

400 centrifuged semen frozen in a soybean lecithin-based extender [21]. Seminal plasma is known to

401 be a main source of antioxidant protection. It is therefore possible that the egg yolk-based semen

402 extender was unable to provide sufficient antioxidant protection to support sperm progressive

403 motility after centrifugation and cooling in the absence of seminal plasma.

404 The initial concentration of cell suspensions also influences sedimentation rate [15]. Density

405 and viscosity of the medium may be influenced not only by the semen extender used but also by

406 the amount of seminal plasma in the ejaculate, the ratio of semen: extender used or the sperm

407 concentration in the suspension being centrifuged. In this study, sperm yield was affected by the

408 concentration at which semen was centrifuged. Centrifugation at a high sperm concentration

$409\left(100 \times 10^{6} / \mathrm{mL}\right)$ resulted in significant sperm losses compared to non-centrifuged semen. It can 
410 be speculated that this finding resulted from differences in density or viscosity of the medium

411 containing different concentrations of seminal plasma, or cell-to-cell interactions in the more

412 concentrated suspension. The properties of the pellet depend on the number of cells, which

413 determines the size of the pellet, centrifugal force and media composition [22]. An increase in

414 the number of cells results in a larger pellet. The larger the pellets the looser they are [22]. The

415 porosity and intermembrane distance between adjacent cells increase, likely due to repositioning

416 and changing orientation of the cells within a larger multi-layer pellet [22]. The larger pellet with

417 lower cell cohesion may have resulted in more cells aspirated with the supernatant rather than in

418 a decrease in sedimentation rate.

419 In conclusion, sperm survival after centrifugation and cooling was not affected by the

420 centrifugation protocol used. Only centrifugation in VMDZ resulted in a decrease in progressive

421 motility after centrifugation and cooling. When equine semen was centrifuged at 400 to $900 \mathrm{x} \mathrm{g}$

422 for 5 to 10 min diluted to a sperm concentration of 25 to $100 \times 10^{6} / \mathrm{mL}$ in milk- or fractionated

423 milk-based semen extenders, loss of motile or viable sperm resulted from a decrease in

424 sedimentation rate rather than cell death within the pellet. Therefore, centrifugation protocols

425 that improve sedimentation rate are likely to improve recovery of motile and viable sperm. With

426 conventional centrifugation protocols, centrifuging volumes of $\leq 20 \mathrm{~mL}$ in 50 - $\mathrm{mL}$ tubes

427 minimized sperm losses in the supernatant. Due to the large volumes of semen that are often

428 processed, using a lower volume may not be practical in all circumstances. If $40-\mathrm{mL}$ suspensions

429 are used, it may be recommended to increase the centrifugation force to $900 \mathrm{xg}$ for $10 \mathrm{~min}$.

430 When using this volume, force and duration, it may be recommended to centrifuge semen at a

431 sperm concentration of 25 to $50 \times 10^{6} / \mathrm{mL}$ since centrifugation at a higher sperm concentration

432 resulted in significant sperm losses. Both milk- (EZ Mixin) and fractionated milk-based 
433 (INRA96) semen extenders seemed equally suitable for centrifugation of equine semen under the

434 conditions tested in this study. Use of an egg yolk-based semen extender (VMDZ) was not

435 recommended for centrifugation due to a significant loss of motile spermatozoa and decrease in

436 progressive sperm motility after cooling. Because there was an effect of stallion on some

437 variables, the ideal centrifugation protocol may need to be adjusted for some individual stallions.

439 Disclosure statement

440

441 The authors declare that there is no conflict of interest that could be perceived as

442 prejudicing the impartiality of the research reported.

443

444 Acknowledgements

445

The authors thank Joanna Kouba and Bradley Purdue from the Department of Animal

447 Sciences, Kansas State University for providing semen samples. Thanks to Dr. Robert Larson

448 from the Department of Clinical Sciences, Kansas State University for his guidance with the

449 statistical analysis.

450

451 References

452

453 [1] Cochran JD, Amann RP, Froman DP, Pickett BW. Effects of centrifugation, glycerol level, 454 cooling to $5^{\circ} \mathrm{C}$, freezing rate and thawing rate on post-thaw motility of equine sperm.

455 Theriogenology 1984;22:25-38. 
456 [2] Pickett BW, Sullivan JJ, Byers WW, pace MM, Remmega EE. Effect of centrifugation and

457 seminal plasma on motility and fertility of stallion and bull spermatozoa. Fertil Steril

$458 \quad 1975 ; 26: 167-174$.

459 [3] Brinsko SP, Crockett EC, Squires EL. Effect of centrifugation and partial removal of seminal 460 plasma on equine spermatozoa motility after cooling and storage. Theriogenology 2000;54:129461136.

462 [4] Love CC, Brinsko SP, Rigby SL, Thompson JA, Blanchard TL, Varner DD. Relationship of 463 seminal plasma level and extender type to sperm motility and DNA integrity. Theriogenology $464 \quad 2005 ; 63: 1584-1591$.

465 [5] Padilla AW, Foote RH. Extender and centrifugation effects on the motility patterns of slow466 cooled stallion spermatozoa. J Anim Sci 1991;69:3308-3313.

467 [6] Dawson GR, Webb GW, Pruitt JA, Loughin TM, Arns MJ. Effect of different processing 468 techniques on motility and acrosomal integrity of cold-stored stallion spermatozoa. J Equine Vet 469 Sci 2000;20:191-194.

470 [7] Loomis PR. Advanced methods for handling and preparation of stallion semen. Vet Clin 471 North Am Equine Pract 2006;22:663-676.

472 [8] Hoogewijs M, Rijsselarae T, De Vliegher S, Vanhaesebrouck E, De Schauwer C, Govaere J, 473 Thys M, Hoflack G, Van Soom A, de Kruif A. Influence of different centrifugation protocols on 474 equine semen preservation. Theriogenology 2010;74:118-126.

475 [9] Len JA, Jenkins JA, Eilts BE, Paccamonti DL, Lyle SK, Hosgood G. Immediate and delayed 476 (after cooling) effects of centrifugation on equine sperm. Theriogenology 2010;73:225-231. 
477 [10] Ecot P, Decuadro-Hansen G, Delhomme G, Vidament M. Evaluation of a cushioned 478 centrifugation technique for processing equine semen for freezing. Anim Reprod Sci

$479 \quad 2005 ; 89: 245-247$.

480 [11] Knop K, Hoffmann N, Rath D, Sieme H. Effects of cushioned centrifugation technique on 481 sperm recovery and sperm quality in stallions with good and poor semen freezability. Anim 482 Reprod Sci 2005;89:294-297.

483 [12] Volkmann DH, van Zyl D. Fertility of stallion semen frozen in $0.5 \mathrm{~mL}$ straws. J Reprod $484 \quad$ Fertil Suppl 1986;35:143-148.

485 [13] Katkov II, Mazur P. Factors affecting yield and survival of cells when suspensions are 486 subjected to centrifugation. Cell Biochemistry and Biophysics 1999;31:231-245.

487 [14] Katkov II, Matzur P. Influence of centrifugation regimes on motility, yield, and cell 488 associations of mouse spermatozoa. J Androl 1998;19:232-241.

489 [15] Wilson K, Walker JM. Centrifugation techniques. In: Principles and techniques of practical 490 biochemistry, Wilson K, Walker JM (Eds.), Cambridge University Press, 1994, p. 275-286.

491 [16] Kuster C. Sperm concentration determination between hemacytmetric and CASA systems:

492 why they can be different. Theriogenology 2005;64:614-617.

493 [17] Douglas-Hamilton DH, Smith NG, Kuster CE, Vermeiden JPW, Althouse GC. Capillary494 loaded particle fluid dynamics: Effect on estimation of sperm concentration. J Androl $495 \quad 2005 ; 26: 115-122$.

496 [18] Webb GW, Dean MM. Effect of centrifugation technique on post-storage characteristics of 497 stallion spermatozoa. J Equine Vet Sci 2009;29:675-680.

498 [19] Aitken RJ, Clarkson JS. Significance of reactive oxygen species and antioxidants in 499 defining the efficiency of sperm preparation techniques. J Androl 1988;9:367-376. 
500 [20] Varisli O, Uguz C, Agca C, Agca Y. Various physical stress factors on rat sperm motility, 501 integrity of acrosome, and plasma membrane. J Androl 2009;30:75-86.

502 [21] Sariözkan S, Bucak MN, Tuncer PB, Taşdemir U, Kinet H, Ulutaş PA. Effects of different 503 extenders and centrifugation/washing on postthaw microscopic-oxidative stress parameters and 504 fertilizing ability of Angora buck sperm. Theriogenology 2010;73:316-323.

505 [22] Abidor IG, Li LH, Hui SW. Studies of cell pellets: I. Electrical properties and porosity. 506 Biophys J 1994;67:418-426.

508 Table 1. Sperm yields and survival factors after centrifugation of equine semen at different 509 forces (400 or $900 \mathrm{x} \mathrm{g}$ ), duration ( 5 or $10 \mathrm{~min}$ ) and volumes ( 20 or $40 \mathrm{~mL}$ ), and after cooling for $51024 \mathrm{~h} . \mathrm{TY}=$ total yield, $\mathrm{TMY}=$ total motile yield, $\mathrm{PMY}=$ progressively motile yield, $\mathrm{VY}=$ viable 511 yield, SFT = survival factor for total motility, SFP = survival factor for progressive motility, $512 \mathrm{SFV}=$ survival factor for viability, SFT2 $4=$ survival factor for total motility at $24 \mathrm{~h}, \mathrm{SFP} 24=$ 513 survival factor for progressive motility at $24 \mathrm{~h}, \mathrm{SFV} 24=$ survival factor for viability at $24 \mathrm{~h}$. $514{ }^{\mathrm{a}, \mathrm{b}, \mathrm{c}, \mathrm{d}, \mathrm{e}}$ Within a row, values with different superscript differ significantly $(\mathrm{P}<0.0001)$ (Mean \pm 515 SD).

517 Table 2. Sperm yields and survival factors after centrifugation of equine semen at $900 \mathrm{x} g$ for 10 $518 \mathrm{~min}$ in $50-\mathrm{mL}$ tubes at different concentrations $\left(25,50\right.$ and $\left.100 \times 10^{6} / \mathrm{mL}\right)$, and after cooling for $51924 \mathrm{~h} . \mathrm{TY}=$ total yield, $\mathrm{TMY}=$ total motile yield, $\mathrm{PMY}=$ progressively motile yield, $\mathrm{VY}=$ viable 520 yield, $\mathrm{SFT}=$ survival factor for total motility, $\mathrm{SFP}=$ survival factor for progressive motility, $521 \mathrm{SFV}=$ survival factor for viability, SFT2 $4=$ survival factor for total motility at $24 \mathrm{~h}, \mathrm{SFP} 24=$ 
522 survival factor for progressive motility at $24 \mathrm{~h}, \mathrm{SFV} 24=$ survival factor for viability at $24 \mathrm{~h}$.

$523{ }^{\mathrm{a}, \mathrm{b}}$ Within a row, values with different superscript differ significantly $(\mathrm{P}<0.05)$ (Mean $\left.\pm \mathrm{SD}\right)$.

525 Table 3. Sperm yields and survival factors after centrifugation at $900 \mathrm{x}$ g for $10 \mathrm{~min}$ in $50-\mathrm{mL}$ 526 tubes in different semen extenders (EZ mixin, INRA96 and VMDZ), and after cooling for $24 \mathrm{~h}$.

$527 \mathrm{TY}=$ total yield, $\mathrm{TMY}=$ total motile yield, $\mathrm{PMY}=$ progressively motile yield, $\mathrm{VY}=$ viable 528 yield, SFT = survival factor for total motility, $\mathrm{SFP}=$ survival factor for progressive motility, 529 SFV = survival factor for viability, SFT24 = survival factor for total motility at $24 \mathrm{~h}$, SFP24 $=$ 530 survival factor for progressive motility at $24 \mathrm{~h}, \mathrm{SFV} 24=$ survival factor for viability at $24 \mathrm{~h}$.

$531{ }^{\mathrm{a}, \mathrm{b}}$ Within a row, values with different superscript differ significantly $(\mathrm{P}<0.05)($ Mean $\pm \mathrm{SD})$.

533 Fig.1. Simplified diagram of a swinging bucket rotor with the position of the tubes containing 40 $534 \mathrm{~mL}$ (left) and $20 \mathrm{~mL}$ (right) of suspension during centrifugation. The centrifugal field is directed 535 radially outwards from the axis of rotation (arrowhead), and is given by the angular velocity of 536 the rotor and the radial distance of the particle from the axis of rotation. Even though the 537 maximum radial distance (distance to the bottom of the tube, $r_{\max }$ ) is the same, the minimum 538 (distance to the meniscus, $r_{\min }$ ) radial distance at the beginning of centrifugation is greater when 539 the tube is partially filled with $20 \mathrm{~mL}$ of suspension than with $40 \mathrm{~mL}$. 


\begin{tabular}{|c|c|c|c|c|c|c|c|c|c|}
\hline \multirow{4}{*}{ Variable } & \multicolumn{9}{|c|}{ Centrifugation force, duration and volume } \\
\hline & $0 \times g$ & $400 \times g$ & $400 \times g$ & $400 \times g$ & $400 \times g$ & $900 \times g$ & $900 \times g$ & $900 \times g$ & $900 \times g$ \\
\hline & $0 \mathrm{~min}$ & $10 \mathrm{~min}$ & $10 \min$ & $5 \mathrm{~min}$ & $5 \mathrm{~min}$ & $10 \mathrm{~min}$ & $10 \mathrm{~min}$ & $5 \mathrm{~min}$ & $5 \mathrm{~min}$ \\
\hline & $40 \mathrm{~mL}$ & $40 \mathrm{~mL}$ & $20 \mathrm{~mL}$ & $40 \mathrm{~mL}$ & $20 \mathrm{~mL}$ & $40 \mathrm{~mL}$ & $20 \mathrm{~mL}$ & $40 \mathrm{~mL}$ & $20 \mathrm{~mL}$ \\
\hline TY (\%) & $100 \pm 0^{\mathrm{a}}$ & $74.5 \pm 7.6^{b}$ & $95.6 \pm 5^{\mathrm{a}}$ & $47.2 \pm 7.3^{\mathrm{c}}$ & $74.3 \pm 8.6^{b}$ & $91.4 \pm 4.5^{\mathrm{a}}$ & $99.2 \pm 0.8^{\mathrm{a}}$ & $72.6 \pm 9.5^{\mathrm{b}}$ & $93.8 \pm 8.9^{\mathrm{a}}$ \\
\hline TMY (\%) & $100 \pm 0^{\mathrm{a}}$ & $71.9 \pm 13.3^{\mathrm{b}}$ & $97.2 \pm 8.7^{\mathrm{a}}$ & $47.5 \pm 10^{\mathrm{c}}$ & $71.2 \pm 5.9^{\mathrm{b}}$ & $92.9 \pm 9.1^{\mathrm{a}}$ & $96.2 \pm 5.5^{\mathrm{a}}$ & $67.1 \pm 10.6^{\mathrm{b}}$ & $94.9 \pm 12^{\mathrm{a}}$ \\
\hline PMY (\%) & $100 \pm 0^{\mathrm{a}}$ & $72.5 \pm 15.2^{\mathrm{b}}$ & $100.4 \pm 9.1^{\mathrm{a}}$ & $49.1 \pm 11.2^{\mathrm{c}}$ & $74 \pm 5.4^{\mathrm{b}}$ & $91.5 \pm 11.3^{\mathrm{a}}$ & $99.5 \pm 7.4^{\mathrm{a}}$ & $68.8 \pm 12.9^{\mathrm{b}}$ & $95.7 \pm 7.4^{\mathrm{a}}$ \\
\hline VY (\%) & $100 \pm 0^{\mathrm{a}}$ & $71.8 \pm 14^{\mathrm{b}, \mathrm{c}, \mathrm{d}}$ & $86.9 \pm 14.2^{\mathrm{a}, \mathrm{b}}$ & $44.5 \pm 8^{\mathrm{e}}$ & $67.1 \pm 7.6^{\mathrm{d}}$ & $84.4 \pm 19.3^{\mathrm{a}, \mathrm{b}, \mathrm{c}}$ & $92 \pm 18.5^{\mathrm{a}}$ & $69.2 \pm 11^{\mathrm{c}, \mathrm{d}}$ & $87.8 \pm 13.1^{\mathrm{a}, \mathrm{b}}$ \\
\hline SFT (\%) & $100 \pm 0$ & $96.6 \pm 15.3$ & $101.7 \pm 8.5$ & $100.6 \pm 15.9$ & $96.4 \pm 8.4$ & $101.6 \pm 8.2$ & $96.9 \pm 5.3$ & $93.1 \pm 13.6$ & $101.2 \pm 7.8$ \\
\hline SFP (\%) & $100 \pm 0$ & $97.5 \pm 18.4$ & $105.1 \pm 8.8$ & $103.6 \pm 16.7$ & $100.1 \pm 6.6$ & $100.1 \pm 10.4$ & $100.3 \pm 7.3$ & $95.3 \pm 15.9$ & $102.5 \pm 7.9$ \\
\hline SFV $(\%)$ & $100 \pm 0$ & $97.1 \pm 19.6$ & $91 \pm 14.7$ & $95.2 \pm 15.1$ & $91 \pm 12.3$ & $92 \pm 18.5$ & $92.8 \pm 18.9$ & $95.7 \pm 12.4$ & $94.1 \pm 13.9$ \\
\hline SFT24 (\%) & $88.5 \pm 15$ & $92.3 \pm 12.3$ & $92.6 \pm 9.4$ & $90.3 \pm 9.4$ & $85.2 \pm 8.4$ & $88.7 \pm 12.1$ & $87.2 \pm 30.4$ & $89.2 \pm 19.1$ & $90.7 \pm 15.2$ \\
\hline SFP24 (\%) & $86.5 \pm 16.5$ & $89.6 \pm 14.5$ & $91.5 \pm 15.6$ & $82.3 \pm 10.9$ & $84.2 \pm 11.1$ & $89.1 \pm 10.6$ & $86.8 \pm 35$ & $80.9 \pm 13.9$ & $89.8 \pm 16.7$ \\
\hline SFV24 (\%) & $96.4 \pm 5.4$ & $94.7 \pm 14.9$ & $103.4 \pm 17.2$ & $92.5 \pm 10.1$ & $97.3 \pm 11.3$ & $87.1 \pm 11.7$ & $100.9 \pm 15.9$ & $96.3 \pm 10$ & $100 \pm 11.1$ \\
\hline
\end{tabular}

540 


\begin{tabular}{lcccc} 
& \multicolumn{4}{c}{ Sperm concentration $(\mathrm{x} \mathrm{10} / \mathrm{mL})$} \\
\cline { 2 - 5 } Variable & Control & 25 & 50 & 100 \\
\hline TY (\%) & $100 \pm 0^{\mathrm{a}}$ & $91.3 \pm 6.4^{\mathrm{a}, \mathrm{b}}$ & $85.4 \pm 15.7^{\mathrm{a}, \mathrm{b}}$ & $83.8 \pm 10.7^{\mathrm{b}}$ \\
TMY (\%) & $100 \pm 0^{\mathrm{a}}$ & $81.6 \pm 12.5^{\mathrm{a}, \mathrm{b}}$ & $83.8 \pm 20.7^{\mathrm{a}, \mathrm{b}}$ & $81.7 \pm 6.8^{\mathrm{b}}$ \\
PMY (\%) & $100 \pm 0$ & $80.6 \pm 27.8$ & $73.9 \pm 22$ & $76.4 \pm 23.5$ \\
VY (\%) & $100 \pm 0^{\mathrm{a}}$ & $70.6 \pm 3.9^{\mathrm{b}}$ & $65.5 \pm 17.7^{\mathrm{b}}$ & $67.1 \pm 14^{\mathrm{b}}$ \\
SFT (\%) & $100 \pm 0$ & $90.3 \pm 6.6$ & $97.5 \pm 7.7$ & $93.1 \pm 30.8$ \\
SFP (\%) & $100 \pm 0$ & $88.3 \pm 19.9$ & $89.3 \pm 22.6$ & $93.1 \pm 30.8$ \\
SFV (\%) & $100 \pm 0$ & $83.9 \pm 13.9$ & $82.5 \pm 18.9$ & $82.7 \pm 17.7$ \\
SFT24 (\%) & $79.2 \pm 13.9$ & $74.8 \pm 21.1$ & $69.1 \pm 19.5$ & $62.3 \pm 20.7$ \\
SFP24 (\%) & $58.1 \pm 33.2$ & $53.1 \pm 36.3$ & $38.1 \pm 18.4$ & $46.7 \pm 60.4$ \\
SFV24 (\%) & $84.9 \pm 18.7$ & $98.2 \pm 26.4$ & $91.5 \pm 9.1$ & $105.9 \pm 30.2$ \\
\hline
\end{tabular}

542

543 
Semen extender

\begin{tabular}{|c|c|c|c|c|c|c|}
\hline Variable & INRA96 Control & INRA96 Centrifuged & VMDZ Control & VMDZ Centrifuged & EZ Mixin Control & EZ Mixin Centrifuged \\
\hline TY (\%) & $100 \pm 0^{\mathrm{a}}$ & $81.8 \pm 11.3^{b}$ & $100 \pm 0^{\mathrm{a}}$ & $86.7 \pm 17.4^{\mathrm{a}, \mathrm{b}}$ & $100 \pm 0^{\mathrm{a}}$ & $93.5 \pm 2.7^{\mathrm{a}, \mathrm{b}}$ \\
\hline TMY (\%) & $100 \pm 0^{\mathrm{a}}$ & $81.5 \pm 14.9^{\mathrm{a}, \mathrm{b}}$ & $100 \pm 0^{\mathrm{a}}$ & $69.3 \pm 22.6^{\mathrm{b}}$ & $100 \pm 0^{\mathrm{a}}$ & $83.7 \pm 18.4^{\mathrm{a}, \mathrm{b}}$ \\
\hline PMY (\%) & $100 \pm 0^{\mathrm{a}}$ & $86.6 \pm 27.2^{\mathrm{a}, \mathrm{b}}$ & $100 \pm 0^{\mathrm{a}}$ & $63.5 \pm 18.2^{b}$ & $100 \pm 0^{\mathrm{a}}$ & $89.9 \pm 10.1^{\mathrm{a}, \mathrm{b}}$ \\
\hline VY (\%) & $100 \pm 0^{\mathrm{a}}$ & $68.4 \pm 30.6^{\mathrm{a}, \mathrm{b}}$ & $100 \pm 0^{\mathrm{a}}$ & $60.9 \pm 36.5^{\mathrm{b}}$ & $100 \pm 0^{\mathrm{a}}$ & $81.4 \pm 15.3^{\mathrm{a}, \mathrm{b}}$ \\
\hline SFT $(\%)$ & $100 \pm 0$ & $99.6 \pm 11.1$ & $100 \pm 0$ & $85 \pm 19.1$ & $100 \pm 0$ & $90 \pm 17$ \\
\hline SFP $(\%)$ & $100 \pm 0$ & $104.9 \pm 23.1$ & $100 \pm 0$ & $80.9 \pm 20.9$ & $100 \pm 0$ & $93.1 \pm 13.5$ \\
\hline $\operatorname{SFV}(\%)$ & $100 \pm 0$ & $82.4 \pm 31.7$ & $100 \pm 0$ & $76.1 \pm 33.7$ & $100 \pm 0$ & $88.6 \pm 13.1$ \\
\hline SFT24 (\%) & $70.7 \pm 18.9$ & $63 \pm 11.2$ & $71.1 \pm 41.6$ & $44.3 \pm 6.6$ & $69.3 \pm 34.1$ & $54.4 \pm 25.9$ \\
\hline SFP24 (\%) & $64.5 \pm 31.7^{\mathrm{a}, \mathrm{b}}$ & $36.9 \pm 14.2^{\mathrm{a}, \mathrm{b}}$ & $70.8 \pm 56.1^{\mathrm{a}}$ & $21 \pm 10.8^{b}$ & $48 \pm 46.7^{\mathrm{a}, \mathrm{b}}$ & $36.9 \pm 30.7^{\mathrm{a}, \mathrm{b}}$ \\
\hline SFV24 (\%) & $86.4 \pm 13$ & $106.4 \pm 41.8$ & $114.3 \pm 48.5$ & $98.8 \pm 37.5$ & $62.8 \pm 32.1$ & $85.4 \pm 33.4$ \\
\hline
\end{tabular}

544

545

546

547

548 
549 Figure 1.

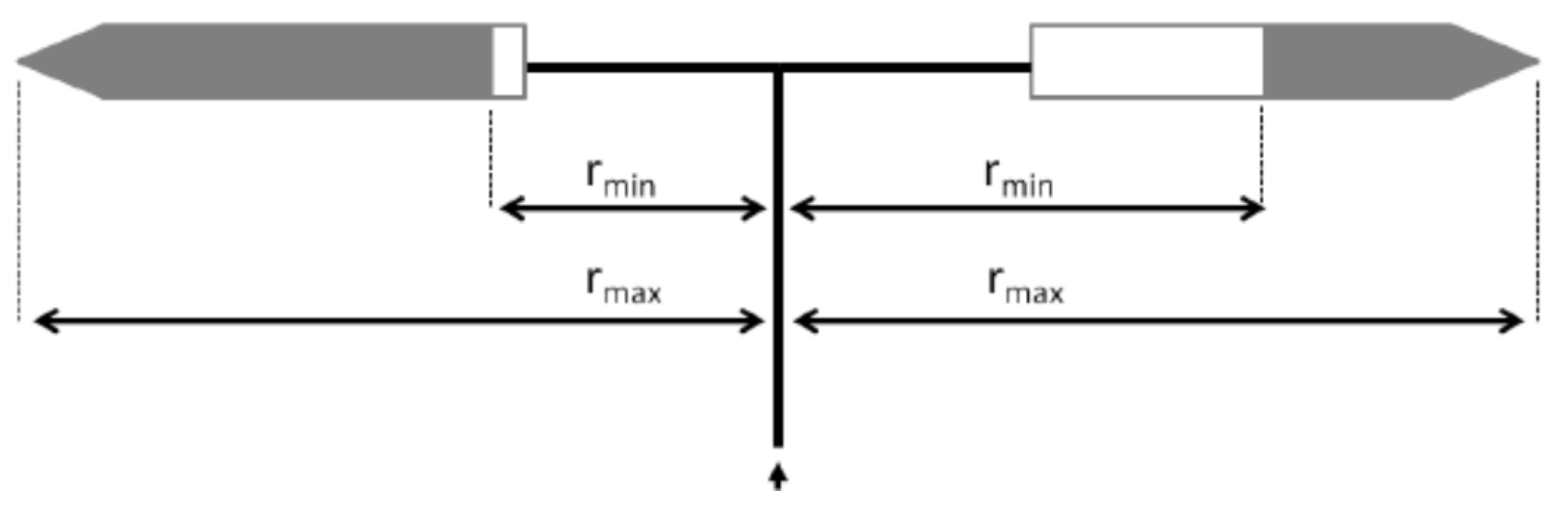

